\title{
On Exceptional Cases of Cauchy Problems for Fuchsian Partial Differential Operators
}

\author{
By \\ Takeshi MANDAI*
}

\begin{abstract}
§0. Introduction
Cauchy problems for Fuchsian partial differential operators have been investigated by several authors, in the space of holomorphic functions ([2], [6], [9]) or in the space of $C^{\infty}$ functions ([8], [10]). To guarantee the unique solvability of the problem, they impose a certain condition, the condition $(C)$ in Proposition 1.1, on the indicial polynomial. This condition is equivalent to that the Taylor expansion of the solution is uniquely determined by the Cauchy data. In the space of $C^{\infty}$ functions, the procedure to solve the Cauchy problem is divided into two steps: First, to find the Taylor expansion of the solution and then to solve the flat Cauchy problem. As for the flat Cauchy problem, the unique solvability is independent of the condition $(C)$. Thus, under the assumption that the flat Cauchy problem is uniquely solvable, we want to consider the case when the condition $(C)$ is not satisfied, by extending the space of admissible solutions.

In this article, for simplicity, we shall consider only Fuchsian equations in the space of $C^{\infty}$ functions. Our arguments are, however, essentially how to get formal solutions, hence similar arguments go well in various other situations: Fuchsian equations in the space of holomorphic functions ([2], [6], [9]), parabolic equations of "Fuchs type" ([1], [7]), etc.

Our program is as follows. In Section 1, we state the main result. After reviewing some basic results on Fuchsian equations in Seetion 2, we prove the main result in Section 3. Finally, in Section 4, we treat Fuchsian systems.
\end{abstract}

\section{§1. Main Result}

Let $\Omega=[0, T] \times \boldsymbol{R}^{n}(T>0)$, where the variable is denoted by $(t, x)$. We

Communicated by S. Matsuura, September 30, 1983.

* Research Institute for Mathematical Sciences, Kyoto University, Kyoto 606, Japan. 
use the notation $\partial_{t}=\partial / \partial t, \partial_{x}=\left(\partial_{x_{1}}, \cdots, \partial_{x_{n}}\right), \partial_{x_{j}}=\partial / \partial x_{j}$. Consider the following operator $(0 \leqq k \leqq m)$.

$$
P=a_{0} t^{k} \partial_{t}^{m}+\sum_{j=1}^{k} a_{j}\left(t, x ; \partial_{x}\right) t^{k-j} \partial_{t}^{m-j}+\sum_{j=k+1}^{m} a_{j}\left(t, x ; \partial_{x}\right) \partial_{t}^{m-j},
$$

where $a_{0}$ is a non-zero constant, $a_{j}\left(t, x ; \partial_{x}\right)(1 \leqq j \leqq m)$ are partial differential operators with respect to $x$ with coefficients in $C^{\infty}(\Omega)$ and ord. $a_{j} \leqq j(1 \leqq j \leqq m)$. We say that $P$ is a Fuchsian differential operator with weight $m-k$, if $a_{j}\left(0, x ; \partial_{x}\right)=a_{j}(x)(1 \leqq j \leqq k)$ are functions of $x$.

We consider the Cauchy problem and the flat Cauchy problem for $P$ :

(C.P.) $\left\{\begin{array}{l}P u=f \quad \text { in } \Omega, \\ \partial_{t}^{j} u_{\mid t=0}=g_{j} \quad \text { in } \boldsymbol{R}^{n}(0 \leqq j \leqq m-k-1) \\ \text { where } f \in C^{\infty}(\Omega) \text { and } g_{j} \in C^{\infty}\left(\boldsymbol{R}^{n}\right)(0 \leqq j \leqq m-k-1) \text { are given. }\end{array}\right.$

(F.C.P.) $\left\{\begin{array}{l}\begin{array}{l}P u=f \quad \text { in } \Omega, \\ u \in C_{f}^{\infty}(\Omega)\end{array} \quad=\left\{\varphi \in C^{\infty}(\Omega) ; \varphi \text { is flat at } t=0\right\} \\ \quad=\left\{\varphi \in C^{\infty}(\Omega) ; \partial_{t}^{j} \varphi_{\mid t=0}=0 \text { for any } j\right\}, \\ \text { where } f \in C_{f}^{\infty}(\Omega) \text { is given. }\end{array}\right.$

We say that (C.P.) (resp. (F.C.P.)) is well-posed, if for any $f \in C^{\infty}(\Omega)$ and any $g_{j} \in C^{\infty}\left(\mathbb{R}^{n}\right)(0 \leqq j \leqq m-k-1)$ (resp. any $\left.f \in C_{f}^{\infty}(\Omega)\right)$, there exists a unique $u \in C^{\infty}(\Omega)$ satisfying (1.2) and (1.3) (resp. (1.4) and (1.5)).

The indicial polynomial of $P$ is

$$
C_{P}(\lambda ; x)=\sum_{j=0}^{k} a_{j}(x) \lambda(\lambda-1) \cdots(\lambda-m+j+1) .
$$

Note that if we write $t^{m-k} P=p\left(t, x ; t \partial_{t}, \partial_{z}\right)$ where $p(t, x ; \lambda, \xi)$ is a polynomial of $(\lambda, \xi)$, then $C_{P}(\lambda ; x)=p(0, x ; \lambda, \xi)$ for any $\xi$. The relation between (C.P.) and (F.C.P.) is as follows. (The proof is given in $\S 2$.)

Proposition 1.1. The Cauchy problem (C.P.) for $P$ is well-posed if and only if the flat Cauchy problem (F.C.P.) is well-posed and P satisfies the following condition.

(C) $C_{P}(\lambda ; x) \neq 0$ for any $x \in \mathbb{R}^{n}$ and any integer $\lambda \geqq m-k$.

Remark 1.2. As is stated in Section 0, the well-posedness of (F.C.P.) for $P$ is independent of the condition (C). For example, the followings hold.

(i) The flat Cauchy problem (F.C.P.) for $P$ is well-posed, if and only if the flat Cauchy problem for another Fuchsian operator $P[z]=t^{-z+m-k} \circ P \circ t^{z}$ is 
well-posed, where $z$ is an arbitrary complex number and o denotes the composition of operators.

(ii) $C_{P[z]}(\lambda ; x)=C_{P}(\lambda+z ; x)$.

From now on, assume the following conditions on $P$.

$$
\left\{\begin{array}{l}
\text { (A.1) The flat Cauchy problem (F.C.P.) for } P \text { is well-posed . } \\
\text { (A.2) There exist integers } m-k \leqq \lambda_{1} \leqq \cdots \leqq \lambda_{s} \text { such that } \\
C_{P}(\lambda ; x)=\prod_{j=1}^{s}\left(\lambda-\lambda_{j}\right) C_{P}(\lambda ; x) \text {, where } C_{P}^{\tilde{P}}(\lambda ; x) \neq 0 \\
\text { for any } x \in \mathbb{R}^{n} \text { and any integer } \lambda \geqq m-k .
\end{array}\right.
$$

We call $\lambda_{1}, \cdots, \lambda_{s}$ the exceptional (characteristic) exponents of $P$.

Since we want to consider solutions including logarithmic terms, we also extend the class of data. Let $L_{h}=\left\{F=\sum_{l=0}^{h} f_{l}(\log t)^{l} ; f_{l} \in C^{\infty}(\Omega)(0 \leqq l \leqq h)\right\}$ and $L=\bigcup_{h=0}^{\infty} L_{h}$. Consider the following Cauchy problem for $P$ :

$$
(\text { C.P. })^{\sim}\left\{\begin{array}{r}
P U=F=\sum_{l=0}^{h} f_{l}(\log t)^{l} \quad \text { in }(0, T] \times \mathbb{R}^{n}, \\
\lim _{t \rightarrow+0} \partial_{t}^{j} U=g_{j} \quad \text { in } \mathbb{R}^{n}(0 \leqq j \leqq m-k-1), \\
\text { where } f_{l} \in C^{\infty}(\Omega)(0 \leqq l \leqq h) \text { and } g_{j} \in C^{\infty}\left(\mathbb{R}^{n}\right) \\
(0 \leqq j \leqq m-k-1) \text { are given }
\end{array}\right.
$$

Now, we state the main result.

Theorem 1.3. (i) For any $f_{l} \in C^{\infty}(\Omega)(0 \leqq l \leqq h)$ and any $g_{j} \in C^{\infty}\left(\mathbb{R}^{n}\right)$ $(0 \leqq j \leqq m-k-1)$, there exist $u_{l} \in C^{\infty}(\Omega)(0 \leqq l \leqq h+s)$ such that

$$
U=u_{0}+\sum_{l=1}^{h} u_{l} t^{m-k}(\log t)^{l}+\sum_{l=1}^{s} u_{h+l} t^{\lambda} l(\log t)^{h+l}
$$

satisfies (1.7) and (1.8). Although this solution is not unique, we can take $\left\{u_{l} ; 0 \leqq l \leqq h+s\right\}$ such that the map $\left(f_{0}, \cdots, f_{h} ; g_{0}, \cdots, g_{m-k-1}\right) \mapsto\left(u_{0}, \cdots, u_{h+s}\right)$ is continuous linear from $C^{\infty}(\Omega)^{h+1} \times C^{\infty}\left(\mathbb{R}^{n}\right)^{m-k}$ into $C^{\infty}(\Omega)^{h+s+1}$.

(ii) Let $\operatorname{Ker}^{\sim} P=\left\{U \in L ; P U=0\right.$ and $\left.\lim _{t \rightarrow+0} \partial_{t}^{j} U=0(0 \leqq j \leqq m-k-1)\right\}$, then $\mathrm{Ker}^{\sim} P$ is parametrized by $C^{\infty}\left(\mathbb{R}^{n}\right)^{s}$ as follows. There exists a closed subspace $K$ of $C^{\infty}(\Omega)^{s}$ such that $K$ is isomorphic to $C^{\infty}\left(\mathbb{R}^{n}\right)^{s}$ and

$$
\operatorname{Ker} \sim P=\left\{U=\sum_{l=1}^{s} u_{l-1} t^{\lambda}(\log t)^{l-1} ;\left(u_{0}, \cdots, u_{s-1}\right) \in K\right\} .
$$

Example 1.4 (Euler-Poisson-Darboux equation). Consider the E-P-D equation: 


$$
\left\{\begin{array}{l}
\left(\partial_{t}^{2}-\Delta_{x}+\frac{k}{t} \partial_{t}\right) u=0 \quad \text { in }(0, T) \times \boldsymbol{R}^{n}, \\
u_{1 t=0}=\varphi(x) \quad \text { in } \boldsymbol{R}^{n}, \\
\partial_{t} u_{1 t=0}=0 \quad \text { in } \boldsymbol{R}^{n},
\end{array}\right.
$$

where $k$ is a non-zero constant and $\Delta_{x}=\sum_{j=1}^{n} \partial_{x_{j}}^{2}$.

We can apply our result to the following modified equation.

$$
\left\{\begin{array}{l}
\left(t \partial_{t}^{2}-t \Delta_{x}+k \partial_{t}\right) u=0 \quad \text { in } \Omega \\
u_{\mid t=0}=\varphi(x) \quad \text { in } \boldsymbol{R}^{n} .
\end{array}\right.
$$

If $k(\neq 0)$ is not a negative integer, then $(C)$ is satisfied and hence for any $\varphi \in C^{\infty}\left(\boldsymbol{R}^{n}\right)$, there exists a unique solution of (1.14) and (1.12). If $k$ is a negative integer, then (1.14) and (1.12) have a solution $u=u_{1}+u_{2} t^{1-k} \log t$, where $u_{1}, u_{2} \in C^{\infty}(\Omega)$. In both cases, (1.13) is necessarily satisfied. Thus, we have a solution of the equations (1.11), (1.12) and (1.13). For the E-P-D equation, many authors have investigated the exceptional cases and constructed solutions explicitly ([3], [4], [5], [11], etc.). They say that the equations (1.11), (1.12) and (1.13) are exceptional only when $k$ is a negative odd integer, because (1.11), (1.12) and (1.13) otherwise have a $C^{\infty}$ solution for any $\varphi \in C^{\infty}\left(\boldsymbol{R}^{n}\right)$. But the case when $k$ is a negative even integer is also "exceptional" in our context. In this case, actually, the solution $u$ of (1.11), (1.12) and (1.13) is not unique, further, there may be no $C^{\infty}$ solution if the right hand side of (1.11) is not zero.

Remark 1.5. The assumption (A.2) seems to be too strong. But, if this condition is not satisfied, then the behavior of solutions of (C.P.) is complicated, as is shown in the following example.

Example 1.6. Consider the operator $P=t \partial_{t}-t \partial_{x}-(t+x)$. The $C^{\infty}$ solutions of $P u=1$ in $(0, T] \times \boldsymbol{R}$ are

$$
u= \begin{cases}\frac{t^{t+x}-1}{t+x}+C(t+x) t^{t+x} & (\text { if } t+x \neq 0), \\ \log t+C(0) & \text { (if } t+x=0),\end{cases}
$$

where $C(s) \in C^{\infty}(R)$ is an arbitrary function. The behavior of this solution $u$ when $t \rightarrow+0$ is very complicated, whatever the function $C$ may be.

In Theorem 1.3, if the data $F$ and $g_{j}(0 \leqq j \leqq m-k-1)$ are "good" data, then there exists a "good" solution. 
Definition 1.7. For an integer $p \geqq m-k$ or $p=\infty$, we say that the data $F \in L_{h}$ and $g_{j} \in C^{\infty}\left(\boldsymbol{R}^{n}\right)(0 \leqq j \leqq m-k-1)$ are $p$-compatible in $L_{h}$ if there exists $U^{\sim} \in L_{h}$ such that $F-P U^{\sim} \in t^{p-m+k} \times L_{h}$ and $\lim _{t \rightarrow+0} \partial_{i}^{j} U^{\sim}=g_{j}(0 \leqq j \leqq m-$ $k-1$ ). (If $p=\infty$, then regard $t^{\infty} \times L_{h}$ as $C_{f}^{\infty}(\Omega)$.) This $U^{\sim}$ is called a $p$-approximate solution (of (C.P.) ) in $L_{h}$. Let $\lambda_{s+1}=\infty$ and let $j(p)=\min \{j \in\{1, \cdots$, $\left.s+1\} ; \lambda_{j} \geqq p\right\}$.

Corollary 1.8. (i) If the data $F \in L_{h}$ and $g_{j} \in C^{\infty}\left(\mathbb{R}^{n}\right)(0 \leqq j \leqq m-k-1)$ are p-compatible in $L_{h}$, then these data are $\lambda_{j(p)}$-compatible in $L_{h}$. Especially, any data are $\lambda_{1}$-compatible.

(ii) Let the data $F \in L_{h}$ and $g_{j} \in C^{\infty}\left(\boldsymbol{R}^{n}\right)(0 \leqq j \leqq m-k-1)$ be p-compatible in $L_{h}$ and let $U^{\sim} \in L_{h}$ be a p-approximate solution. Then, there exist $u_{l} \in C^{\infty}(\Omega)$ $(0 \leqq l \leqq h+s-j(p)+1)$ such that

$$
U=U^{\sim}+\sum_{l=0}^{h} u_{l} t^{p}(\log t)^{l}+\sum_{l=1}^{s-j(p)+1} u_{h+l} t^{\lambda(p)+l-1}(\log t)^{h+l}
$$

satisfies (1.7) and (1.8).

\section{§ 2. Review on Fuchsian Operators}

In this section, we review some basic results on Fuchsian operators. Most of the results in this section are well-known or elementary, and their proofs are omitted.

Let $P$ be a Fuchsian operator defined by (1.1). First, consider the equation $P u=f$ for $u, f \in C^{\infty}(\Omega)$.

Lemma 2.1. For $P$, there exist $B_{j}\left(\lambda ; x, \partial_{x}\right)(j=1,2, \cdots)$, which are differential operators on $\boldsymbol{R}^{n}$ and polynomials with respect to $\lambda$, such that the equations $\partial_{t}^{j}(P u)_{\mid t=0}=\partial_{t}^{j} f_{\mid t=0}(j=0,1, \cdots)$ are equivalent to

$$
\begin{gathered}
C(j ; x) u_{j}=f_{j-m+k}-\sum_{i=1}^{j} B_{i}\left(j-i ; x, \partial_{x}\right) u_{j-i} \\
(j=m-k, m-k+1, \cdots)
\end{gathered}
$$

where $\sum_{j=0}^{\infty} u_{j} t^{j}$ (resp. $\left.\sum_{j=0}^{\infty} f_{j} t^{j}\right)$ is the Taylor expansion of $u$ (resp. $f$ ).

From this lemma, we get Proposition 1.1.

Proof of Proposition 1.1. (I) Assume that (C.P.) for $P$ is well-posed. In (2.1), for any $\left\{f_{j} ; j=0,1, \cdots\right\}$ and any $\left\{u_{0}, \cdots, u_{m-k-1}\right\}$, there exists $\left\{u_{j} ; j\right.$ $=m-k, m-k+1, \cdots\}$. Hence, $P$ satisfies the condition (C). Let $f \in C_{f}^{\infty}(\Omega)$. 
There exists a unique solution $u$ of (C.P.) for $g_{j}=0(0 \leqq j \leqq m-k-1)$. By (2.1), this solution $u$ belongs to $C_{f}^{\infty}(\Omega)$. Thus, the flat Cauchy problem (F.C.P.) for $P$ is well-posed.

(II) Assume that (F.C.P.) for $P$ is well-posed and that $P$ satisfies (C). Let $f \in C^{\infty}(\Omega)$ and $g_{j} \in C^{\infty}\left(\boldsymbol{R}^{n}\right)(0 \leqq j \leqq m-k-1)$. The system of equations (2.1) has a unique solution $\left\{u_{j} ; j=0,1, \cdots\right\}$, where $j ! u_{j}=g_{j}(0 \leqq j \leqq m-k-1)$. There exists $v \in C^{\infty}(\Omega)$ such that $\sum_{j=0}^{\infty} u_{j} t^{j}$ is the Taylor expansion of $v$. Noting that $P v-f \in C_{f}^{\infty}(\Omega)$, there exists a solution $w \in C_{f}^{\infty}(\Omega)$ of $P w=f-P v$, and $u=v+w$ is a solution of (C.P.). On the other hand, if $P u=0$ and $g_{j}=0(0 \leqq j$ $\leqq m-k-1$ ), then $u \in C_{f}^{\infty}(\Omega)$ by (2.1), hence $u=0$. Thus, (C.P.) for $P$ is wellposed.

Definition 2.2. (i) For $P$, we can write $\hat{P}=t^{m-k} P=p\left(t, x ; t \partial_{t}, \partial_{x}\right)$, where $p(t, x ; \lambda, \xi)$ is a polynomial of $(\lambda, \xi)$. Put $\hat{P}[z]=t^{-z} \circ \hat{P} \circ t^{z}=p\left(t, x ; t \partial_{t}+z, \partial_{x}\right)$ for a complex number $z$ and put $P_{l}=p_{l}\left(t, x ; t \partial_{t}, \partial_{x}\right)$ where $p_{l}(t, x ; \lambda, \xi)$ $=\partial_{\lambda}^{l} p(t, x ; \lambda, \xi)$. Note that $C_{P[z]}(\lambda ; x)=C_{P}(\lambda+z ; x)$ and $C_{P_{l}}(\lambda ; x)=\partial_{\lambda}^{l} C_{P}(\lambda ; x)$. (ii) Put $F\langle k\rangle=C^{\infty}(\Omega) /\left(t^{k} \times C^{\infty}(\Omega)\right) \cong \bigoplus_{l=0}^{k-1} C^{\infty}\left(\mathbb{R}^{n}\right) t^{l}$. We can regard $\hat{P}$ as an operator on $F\langle k\rangle(k=0,1, \cdots)$.

Lemma 2.3. (i) For any $v \in C^{\infty}\left((0, T) \times \boldsymbol{R}^{n}\right)$, there hold the following formulas.

$$
\left\{\begin{array}{l}
\hat{P}\left(t^{z} v\right)=t^{z} P[z](v), \\
\hat{P}\left(v(\log t)^{h}\right)=\sum_{l=0}^{h}\left(\begin{array}{l}
h \\
l
\end{array}\right)\left(P_{l} v\right)(\log t)^{h-l},
\end{array}\right.
$$

where $h$ is a non-negative integer and $\left(\begin{array}{l}h \\ l\end{array}\right)=\frac{h !}{l !(h-l) !}$.

(ii) If $k=m$, then the operator $P: C^{\infty}(\Omega) \rightarrow C^{\infty}(\Omega)$ is an isomorphism modulo $t^{\lambda_{1}}$. In other words, the operator $P: F\left\langle\lambda_{1}\right\rangle \rightarrow F\left\langle\lambda_{1}\right\rangle$ is an isomorphism.

(iii) If $k=m, \lambda_{1}=\cdots=\lambda_{r}=0<\lambda_{r+1}$, then as operators on $F\langle 1\rangle$, we have $P_{l}=0(l=0,1, \cdots, r-1)$ and $P_{r}$ is an isomorphism. In other words, $\left(P_{l} u\right)_{\mid t=0}=0$ $(0 \leqq l \leqq r-1)$ and $\left(P_{r} u\right)_{\mid t=0}=c(x)\left(u_{\mid t=0}\right)$ for any $u \in C^{\infty}(\Omega)$, where $c \in C^{\infty}\left(\mathbb{R}^{n}\right)$ and $c(x) \neq 0$ for any $x \in \mathbb{R}^{n}$.

The representation $U=\sum_{l=0}^{H} u_{l}(\log t)^{l}$ of $U \in L_{H}$ is not unique, but the following holds.

Lemma 2.4. Let $u_{l} \in C^{\infty}\left(\boldsymbol{R}^{n}\right)(0 \leqq l \leqq H), U=\sum_{l=0}^{H} u_{l}(\log t)^{l}$ and let $d$ be $a$ 
non-negative integer. Then, there holds $\lim _{t \rightarrow+0} \partial_{t}^{j} U=0(0 \leqq j \leqq d)$ if and only if $\partial_{t}^{j} u_{l \mid t=0}=0(0 \leqq j \leqq d, 0 \leqq l \leqq H)$.

Finally, we state the reduction procedure of (C.P.) $)^{\sim}$ for $P$ to (C.P. $)^{\sim}$ for another Fuchsian operator $P[m-k]$ with weight 0 .

Proposition 2.5. For the initial data $\left(g_{0}, \cdots, g_{m-k-1}\right)$ in $(\text { C.P. })^{\sim}$ for $P$, put $G=\sum_{j=0}^{m-k-1} \frac{1}{j !} g_{j} t^{j}$. For $U \in L$, the initial condition $\lim _{t \rightarrow+0} \partial_{t}^{j} U=g_{j}(0 \leqq j \leqq m-k-1)$ is equivalent to that $U=G+t^{m-k} U^{\sim}$ for some $U^{\sim} \in L$. Putting $F^{\sim}=F-P G$, we can reduce $(C . P .)^{\sim}$ for $P$ to the equation $P[m-k] U^{\sim}=F^{\sim}$. Especially, the Cauchy problem (C.P.) for $P$ is well-posed if and only if the Cauchy problem for $P[m-k]$ is well-posed.

If $P$ satisfies the conditions (A.1), (A.2), then $P[m-k]$ also satisfies the same conditions. Further, the exceptional exponents of $P[m-k]$ are $\lambda_{1}-m+k$, $\cdots, \lambda_{s}-m+k$. Thus, we have only to consider the case with weight 0 . In this case, for $U=\sum_{l=0}^{H} u_{l}(\log t)^{l}(h \leqq H)$, we have $P u=\sum_{l=0}^{H}\left\{\sum_{j=0}^{H-l}\left(\begin{array}{c}l+j \\ j\end{array}\right)\left(P_{j} u_{l+j}\right)\right\}(\log t)^{l}$ by Lemma 2.3 (i). Therefore, we consider the system of equations:

$$
P_{0} u_{l}+\cdots+\left(\begin{array}{c}
l+j \\
j
\end{array}\right) P_{j} u_{l+j}+\cdots+\left(\begin{array}{c}
H \\
H-l
\end{array}\right) P_{H-l} u_{H}=f_{l} \quad(0 \leqq l \leqq H)
$$

where $f_{l}=0$ if $l>h$.

\section{§3. Proof of Main Result}

We may assume that $P$ has weight 0 , as is shown in the previous section. First, we prove Theorem 1.3 by the induction on $s$.

Proof of Theorem 1.3. (I) The case $s=0$. If $s=0$, then $P$ satisfies the condition (C), hence (C.P.) for $P$ is well-posed. By the system of equations (2.4) for $H=h$, the solution $\left\{u_{l} ; l=0,1, \cdots, h\right\}$ is uniquely determined from $\left\{f_{l} ; l=0\right.$, $1, \cdots, h\}$. Conversely, if $U=\sum_{l=0}^{H} u_{l}(\log t)^{l}, u_{l} \in C^{\infty}(\Omega)$ and $P U=0$, then by Lemma 2.4, the system of equations (2.4) holds for some $f_{l} \in C_{f}^{\infty}(\Omega)(0 \leqq l \leqq H)$. By Lemma 2.1, we have $u_{l} \in C_{f}^{\infty}(\Omega)(0 \leqq l \leqq H)$, hence $U \in C_{f}^{\infty}(\Omega)$. By (A.1), we have $U=0$. Thus, Theorem 1.3 holds if $s=0$.

(II) Reduction to the case $\lambda_{1}=0$. Let $s \geqq 1$. By Lemma 2.3 (ii), the system of equations (2.4) is uniquely solvable in $F\left\langle\lambda_{1}\right\rangle$. That is, we have $\left(\partial_{t}^{j} u_{l}\right)_{\mid t=0}=0$ $\left(l \geqq h+1,0 \leqq j \leqq \lambda_{1}-1\right)$, furthermore the functions $\left(\partial_{t}^{j} u_{l}\right)_{\mid t=0}\left(l \leqq h, 0 \leqq j \leqq \lambda_{1}-1\right)$ 
are determined by $\left\{f_{l} ; l=0,1, \cdots, h\right\}$. Put $U^{\sim}=\sum_{l=0}^{h} \sum_{j=0}^{\lambda_{1}-1} \frac{1}{j !}\left(\partial_{t}^{j} u_{l}\right)_{\mid t=0} t^{j}(\log t)^{l}$, then $F-P U^{\sim}=t^{\lambda_{1} F^{\sim}}$, for some $F^{\sim}=\sum_{l=0}^{h} f_{l}^{\sim}(\log t)^{l}, f_{l}^{\sim} \in C^{\infty}(\Omega) . \quad$ By $U=U^{\sim}$ $+t^{\lambda_{1}} V$, the equation $P U=F$ is reduced to $P\left[\lambda_{1}\right] V=F^{\sim}$. Since we can take $\left\{f_{\tilde{l}} ; l=0,1, \cdots, h\right\}$ such that the map $\left(f_{0}, \cdots, f_{h}\right) \mapsto\left(f_{0}, \cdots, f_{\tilde{h}}\right)$ is continuous linear, we may assume that $\lambda_{1}=0$.

(III) The case $\lambda_{1}=0$. Let $\lambda_{1}=\cdots=\lambda_{r}=0<\lambda_{r+1}(r \leqq s)$. Considering (2.4) modulo $t$, we have $\left(u_{H}\right)_{\mid t=0}=\left(u_{H-1}\right)_{\mid t=0}=\cdots=\left(u_{r+h+1}\right)_{\mid t=0}=0$ and the functions $\left(u_{r+h}\right)_{\mid t=0}, \cdots,\left(u_{r}\right)_{\mid t=0}$ are uniquely determined from $\left\{f_{l} ; 0 \leqq l \leqq h\right\}$ by Lemma 2.3 (iii). Put $W=\sum_{l=0}^{h}\left(u_{r+l}\right)_{\mid t=0}(\log t)^{r+l}$, then $F-P W=t F^{\sim}$ for some $F^{\sim}$ $=\sum_{l=0}^{r+h} f \tilde{l}(\log t)^{l} \in L_{r+h}$. Putting $U=W+t V$, the equation $P U=F$ is reduced to $P[1] V=F^{\sim}$. We can take $\left\{f_{l} ; l=0,1, \cdots, r+h\right\}$ such that the map $\left(f_{0}, \cdots, f_{h}\right)$ $\mapsto\left(f_{0}, \cdots, f_{r+h}\right)$ is continuous linear. Further, the exceptional exponents of $P[1]$ are $\lambda_{r+1}-1, \cdots, \lambda_{s}-1$. Thus, by the induction hypothesis, we get (i).

To prove (ii), let $f_{l} \in C_{f}^{\infty}(\Omega)$ for any $l$. In (2.4), we have $u_{H \mid t=0}=\cdots$ $=u_{r \mid t=0}=0$. For any $\alpha_{0}, \cdots, \alpha_{r-1} \in C^{\infty}\left(\boldsymbol{R}^{n}\right)$, put $G=\sum_{l=0}^{r-1} \alpha_{l}(\log t)^{l}$. There holds $P G=t F$ for some $F \in L_{r-1}$ by Lemma 2.3 (iii). Putting $U=G-t V$, the equation $P U=0$ is equivalent to $P[1] V=F$. By the result of (i), this equation has a solution $V=\sum_{l=0}^{r-1} v_{l}(\log t)^{l}+\sum_{l=1}^{s-r} v_{r+l-1} t^{\lambda_{r+l^{-1}}}(\log t)^{r+l-1}$, where $v_{l} \in C^{\infty}(\Omega)(0 \leqq l \leqq$ $s-1)$. We can take this solution such that the map $\left(\alpha_{0}, \cdots, \alpha_{r-1}\right) \mapsto\left(v_{0}, \cdots, v_{s-1}\right)$ is continuous linear. For $0 \leqq l \leqq s-1$, let $S_{l}$ be the map from $C^{\infty}\left(\boldsymbol{R}^{n}\right)^{r}$ into $C^{\infty}(\Omega)$ given by $S_{l}\left(\alpha_{0}, \cdots, \alpha_{r-1}\right)=\alpha_{l}-v_{l} t(0 \leqq l \leqq r-1)$ and $=-v_{l}(r \leqq l \leqq s-1)$. By the induction hypothesis, there exists an isomorphism $T^{\sim}$ from $C^{\infty}\left(\boldsymbol{R}^{n}\right)^{s-r}$ into $C^{\infty}(\Omega)^{s-r}$ such that $\operatorname{Ker}^{\sim} P[1]=\left\{W=\sum_{l=1}^{s-r} T \tilde{l}_{l-1}(\beta) t^{\lambda_{r+l}-1}(\log t)^{l-1} ; \beta \in C^{\infty}\left(\boldsymbol{R}^{n}\right)^{s-r}\right\}$, where $T^{\sim}(\beta)=\left(T_{0}^{\sim}(\beta), \cdots, T_{\tilde{s}-r-1}^{\tilde{1}}(\beta)\right)$. For $0 \leqq l \leqq s-1$, let $T_{l}$ be the map from $C^{\infty}\left(\boldsymbol{R}^{n}\right)^{s}$ into $C^{\infty}(\Omega)$ given by $T_{l}\left(\alpha_{0}, \cdots, \alpha_{r-1} ; \beta\right)=S_{l}\left(\alpha_{0}, \cdots, \alpha_{r-1}\right)-$ $T \tilde{l}(\beta) t^{\lambda_{r+l+1}-\lambda_{l+1}}$, where $T \tilde{l}(\beta)=0$ if $l \geqq s-r$. Note that $T_{l}\left(\alpha_{0}, \cdots, \alpha_{r-1} ; \beta\right)_{\mid t=0}$ $=\alpha_{l}(0 \leqq l \leqq r-1)$. It is easy to see that the map $T=\left(T_{0}, \cdots, T_{s-1}\right)$ is an isomorphism from $C^{\infty}\left(\boldsymbol{R}^{n}\right)^{s}$ into $C^{\infty}(\Omega)^{s}$. Thus, we get (ii).

Remark 3.1. Our solutions obtained above have the following property: If $f_{l} \in C_{f}^{\infty}(\Omega)(0 \leqq l \leqq h)$ and $g_{j}=0(0 \leqq j \leqq m-k-1)$, then $u_{l} \in C_{f}^{\infty}(\Omega)(0 \leqq l$ $\leqq h+s)$.

Next, we prove Corollary 1.8 . 
Proof of corollary 1.8. Let $F-P U^{\sim}=t^{p} F^{\sim}$, where $F^{\sim} \in L_{h}$. Putting $U=U^{\sim}+t^{p} V$, the equation $P U=F$ is reduced to the equation $P[p] V=F^{\sim}$. Since the exceptional exponents of $P[p]$ are $\lambda_{j(p)}-p, \cdots, \lambda_{s}-p$, this equation has a solution $V=\sum_{l=0}^{h} u_{l}(\log t)^{l}+\sum_{l=1}^{s-j(p)+1} u_{h+l} t^{\lambda}{ }_{j(p)+l-1}^{-p}(\log t)^{h+l}$ by Theorem 1.3 (i). Therefore, we get (ii).

In the solution obtained in (ii), put $U_{0}=U^{\sim}+\sum_{l=0}^{h} u_{l} t^{p}(\log t)^{l}$. Then, $P U_{0}-F \in t^{\lambda_{j(p)}} \times L$, hence $U_{0}$ is a $\lambda_{j(p)}$-approximate solution of (C.P.) $\sim$ in $L_{h}$. Thus, we get (i).

\section{§4. Fuchsian Systems}

In this section, we consider Fuchsian systems. Consider the operator $Q=t \partial_{t} I_{N}-A\left(t, x ; \partial_{x}\right)$, where $I_{N}$ is $(N \times N)$ unit matrix and $A\left(t, x ; \partial_{x}\right)$ $=\left(a_{i, j}\left(t, x ; \partial_{x}\right)\right)_{1 \leqq i, j \leqq N}$. Assune that $a_{i, j}\left(t, x ; \partial_{x}\right)$ are differential operators with respect to $x$ with coefficients in $C^{\infty}(\Omega)$ and that $a_{i, j}\left(0, x ; \partial_{x}\right)$ are functions of $x$. Put $A_{0}(x)=A\left(0, x ; \partial_{x}\right)$. (We make no assumption on the order of $a_{i, j}\left(t, x ; \partial_{x}\right)$.) Consider the Cauchy problem and the flat Cauchy problem for $Q$.

$$
\begin{aligned}
& \text { (C.P.) }\left\{\begin{array}{l}
Q u=f \quad \text { in } \Omega, \\
\text { where } f \in C^{\infty}(\Omega)^{N} \text { is given . }
\end{array}\right. \\
& \text { (F.C.P.) }\left\{\begin{array}{l}
Q u=f \quad \text { in } \Omega, \\
u \in C_{f}^{\infty}(\Omega)^{N}, \\
\text { where } f \in C_{f}^{\infty}(\Omega)^{N} \text { is given . }
\end{array}\right.
\end{aligned}
$$

We make the following assumptions on $Q$.

(A.3) The flat Cauchy problem (F.C.P.) for $Q$ is well-posed.

(A.4) There exist non-negative integers $0 \leqq \mu_{1}<\mu_{2}<\cdots<\mu_{d}$ and positive integers $r_{1}, r_{2}, \cdots, r_{d}$ such that $\operatorname{det}\left(\lambda I_{N}-A_{0}(x)\right)=\prod_{j=1}^{d}\left(\lambda-\mu_{j}\right)^{r} C^{\sim}(\lambda ; x)$, where $C^{\sim}(\lambda ; x) \neq 0$ for any $x \in \boldsymbol{R}^{n}$ and any integer $\lambda \geqq 0$.

Now, for a fixed $x \in \boldsymbol{R}^{n}$, let the Jordan's normal form of $A_{0}(x)$ be

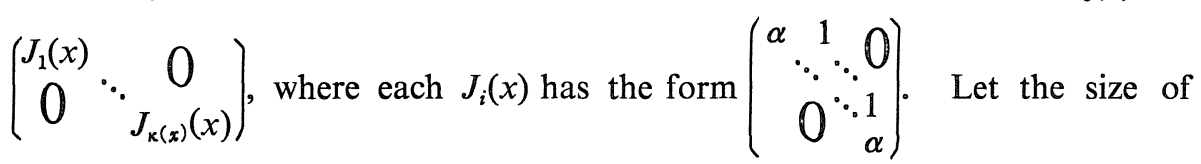
$J_{i}(x)$ be $\sigma_{i}(x)$. For $1 \leqq j \leqq d$, put $m_{j}=\max \left\{\sigma_{i}(x) ; x \in \mathbb{R}^{n}, 1 \leqq i \leqq \kappa(x)\right.$, the 
eigenvalue of $J_{i}(x)$ is $\left.\mu_{j}\right\}$. In other words, $m_{j}=\min \left\{m\right.$; the rank of $\left(\mu_{j} I_{N}\right.$ $\left.-A_{0}(x)\right)^{m}$ is $N-r_{j}$ for any $\left.x \in \mathbb{R}^{n}\right\}$. Put $\lambda_{j}=\mu_{k}$ if $\sum_{j=1}^{k-1} m_{j}+1 \leqq j \leqq \sum_{j=1}^{k} m_{j}(1 \leqq k$ $\leqq d)$ and put $s=\sum_{j=1}^{d} m_{j}$. Let $r=\sum_{j=1}^{d} r_{j}$ and $r_{0}=N-r$.

We consider the following problem for $Q$.

$$
\text { (C.P.) }\left\{\begin{array}{l}
Q U=F=\sum_{l=0}^{h} f_{l}(\log t)^{l} \quad \text { in }(0, T] \times \mathbb{R}^{n}, \\
\text { where } f_{l} \in C^{\infty}(\Omega)^{N}(0 \leqq l \leqq h) \text { are given. }
\end{array}\right.
$$

Theorem 4.1. (i) For any $f_{l} \in C^{\infty}(\Omega)^{N}(0 \leqq l \leqq h)$, there exist $u_{l} \in C^{\infty}(\Omega)^{N}$ $(0 \leqq l \leqq h+s)$ such that

$$
U=u_{0}+\sum_{l=1}^{h} u_{l}(\log t)^{l}+\sum_{l=1}^{s} u_{h+l} t^{\lambda_{l}}(\log t)^{h+l}
$$

satisfies (4.4). Further, we can take $\left\{u_{l} ; l=0,1, \cdots, h+s\right\}$ such that the map $\left(f_{0}, \cdots, f_{h}\right) \mapsto\left(u_{0}, \cdots, u_{h+s}\right)$ is continuous linear from $C^{\infty}(\Omega)^{N \times(h+1)}$ into $C^{\infty}(\Omega)^{N \times(h+s+1)}$.

(ii) Let $\operatorname{Ker}^{\sim} Q=\left\{U \in L^{N} ; Q U=0\right\}$, then $\operatorname{Ker}^{\sim} Q$ is parametrized by $C^{\infty}\left(\boldsymbol{R}^{n}\right)^{r}$ as follows. There exists a closed subspace $K$ of $C^{\infty}(\Omega)^{N \times s}$, isomorphic to $C^{\infty}\left(\boldsymbol{R}^{n}\right)^{r}$, such that

$$
\begin{gathered}
\operatorname{Ker}^{\sim} Q=\left\{U=\sum_{l=1}^{s} u_{l-1} t^{\lambda}{ }_{l}(\log t)^{l-1} ; u_{l} \in C^{\infty}(\Omega)^{N},\right. \\
\left.\left(u_{0}, \cdots, u_{s-1}\right) \in K\right\} .
\end{gathered}
$$

We begin the proof of Theorem 4.1 by the following lemma.

Lemma 4.2. There exist $C^{\infty}(N \times N)$-matrix $S(x)$ and $C^{\infty}\left(r_{j} \times r_{j}\right)$-matrix $B_{j}(x)(0 \leqq j \leqq d)$ such that

(i) $S(x)^{-1}$ exists,

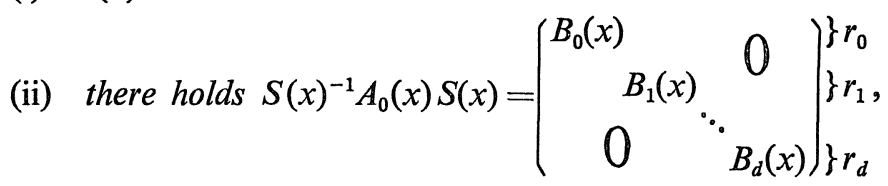

(iii) for $1 \leqq j \leqq d, B_{j}(x)$ has only one eigenvalue $\mu_{j}$,

(iv) no eigenvalue of $B_{0}(x)$ is a non-negative integer.

The proof of this lemma is left to readers. Note that $\left(\mu_{j} I_{r_{j}}-B_{j}(x)\right)^{m_{j}}=0$ $(1 \leqq j \leqq d)$ by the definition of $m_{j}$.

We have the similar results as Proposition 1.1, Lemma 2.1, 2.3.

Lemma 4.3. (i) There exist matrices of differential operators $A_{i}\left(x, \partial_{x}\right)$ 
$(i=1,2, \cdots)$ such that the equations $\partial_{t}^{j}(Q u)_{\mid t=0}=\partial_{t}^{j} f_{\mid t=0}(j=0,1, \cdots)$ are equivalent to

$$
\left\{j I_{N}-A_{0}(x)\right\} u_{j}=f_{j}+\sum_{i=1}^{j} A_{i}\left(x ; \partial_{x}\right) u_{j-i} \quad(j=0,1, \cdots),
$$

where $\sum_{j=0}^{\infty} u_{j} t^{j}$ (resp. $\sum_{j=0}^{\infty} f_{j} t^{j}$ ) is the Taylor expansion of $u$ (resp. $f$ ).

(ii) The Cauchy problem (C.P.) for $Q$ is well-posed if and only if the flat Cauchy problem (F.C.P.) for $Q$ is well-posed and (A.4) for $d=0$ holds.

(iii) For any $v \in C^{\infty}\left((0, T] \times \mathbb{R}^{n}\right)^{N}$, there hold the followings.

$$
\left\{\begin{array}{l}
Q\left(t^{k} v=t^{k} Q[k] v\right. \\
Q\left(v(\log t)^{l}\right)=(Q v)(\log t)^{l}+l v(\log t)^{l-1}
\end{array}\right.
$$

where $Q[k]=t \partial_{t} I_{N}+k I_{N}-A\left(t, x ; \partial_{x}\right)$.

(iv) Let $F\langle k\rangle=C^{\infty}(\Omega)^{N} /\left(t^{k} \times C^{\infty}(\Omega)\right)^{N}$. The operator $Q$ can be regarded as an operator on $F\langle k\rangle$. The operator $Q$ is an isomorphism on $F\left\langle\mu_{1}\right\rangle$ and $Q=-A_{0}(x)$ on $F\langle 1\rangle \cong C^{\infty}\left(\mathbb{R}^{n}\right)^{N}$.

By (iii) in the above lemma, we have $Q\left(\sum_{l=0}^{H} u_{l}(\log t)^{l}\right)=\sum_{l=0}^{H}\left(Q u_{l}+(l+1) \times\right.$ $\left.u_{l+1}\right)(\log t)^{l}$, where $u_{H+1}=0$. Therefore, we consider a system of equations

$$
Q u_{l}+(l+1) u_{l+1}=f_{l} \quad(0 \leqq l \leqq H),
$$

where $u_{H+1}=f_{h+1}=\cdots=f_{H}=0$. We shall prove Theorem 4.1 by the induction on $d$.

Proof of Theorem 4.1. We may assume that $A_{0}(x)=\left(\begin{array}{ccc}B_{0}(x) & & 0 \\ 0 & \ddots & 0 \\ & & B_{d}(x)\end{array}\right)$, where $B_{j}(x)(0 \leqq j \leqq d)$ have the properties stated in Lemma 4.2.

(I) The case $d=0$. If $d=0$, then Theorem 4.1 holds by the argument similar to that in $(\mathbb{I})$ of the proof of Theorem 1.3.

(II) Reduction to the case $\mu_{1}=0$. Let $d \geqq 1$. We may assume that $\mu_{1}=0$, by the argument similar to that in (II) of the proof of Theorem 1.3.

(III) The case $\mu_{1}=0$. Let $\mu_{1}=0$. If $u_{0}$ is given, then $u_{1}, \cdots, u_{H}$ are uniquely determined by (4.8) and the system of equations (4.8) is equivalent to the single equation

$$
Q^{H+1} u_{0}+\sum_{l=0}^{h}(-1)^{l+1} l ! Q^{H-l} f_{l}=0 .
$$

Take $H=m_{1}+h$ and consider (4.9) modulo $t$, that is,

$$
A_{0}(x)^{m_{1}+h+1}\left(u_{0 \mid t=0}\right)=-\sum_{l=0}^{h} l ! A_{0}(x)^{m_{1}+h-l}\left(f_{l \mid t=0}\right) .
$$


Since $A_{0}(x)^{j}=\left(\begin{array}{ccccc}B_{0}(x)^{j} & & & & \\ & 0 & & 0 \\ & & B_{2}(x)^{j} & & \\ 0 & & & \ddots & \\ & & & B_{d}(x)^{j}\end{array}\right)$ for $j \geqq m_{1}$ and $B_{k}(x)^{j}$ is invertible for $k \neq 1$, the equation (4.10) has a solution $u_{0 \mid t=0}$. If we take $u_{0 \mid t=0}$ in the form

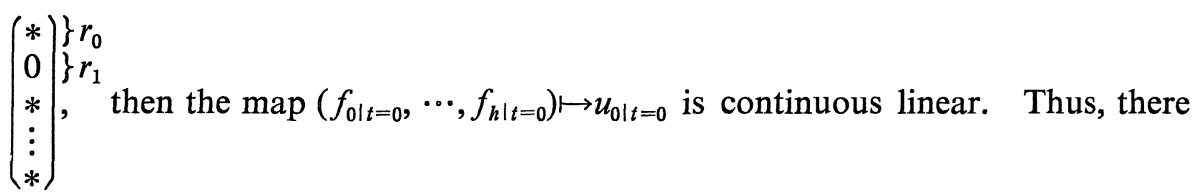
exist $v_{j} \in C^{\infty}\left(\boldsymbol{R}^{n}\right)^{N} \quad\left(0 \leqq j \leqq m_{1}+h\right)$ such that $V=\sum_{j=0}^{m_{1}+h} v_{j}(x)(\log t)^{j}$ satisfies $Q V-F=t F^{\sim}$ for some $F^{\sim}=\sum_{l=0}^{m_{1}+h} f_{l}(\log t)^{l}, f_{l} \in C^{\infty}(\Omega)^{N} \quad(0 \leqq l \leqq h)$. Putting $U=V+t W$, the equation $Q U=F$ is reduced to $Q[1] W=F^{\sim}$. Since we can take $\left\{f_{l} ; l=0,1, \cdots, m_{1}+h\right\}$ such that the map $\left(f_{0}, \cdots, f_{h}\right) \mapsto\left(f_{0}, \cdots, f_{m_{1}+h}\right)$ is continuous linear, we get (i) by the induction hypothesis.

To prove (ii), consider (4.9) modulo $t$ for $H=h$ and $f_{l}=0(0 \leqq l \leqq H=h)$. That is, $A_{0}(x)^{H+1}\left(u_{0 \mid t=0}\right)=0$. If $H+1 \geqq m_{1}$, this is equivalent to that there holds $u_{0 \mid t=0}=\left(\begin{array}{c}0 \\ \alpha(x) \\ 0 \\ \vdots \\ 0\end{array}\right)^{\}}$for some $\alpha \in C^{\infty}\left(\boldsymbol{R}^{n}\right)^{r} r_{1}$. For any $\alpha \in C^{\infty}\left(\boldsymbol{R}^{n}\right)^{r}$, put $u_{0 \mid t=0}$ as above, then $u_{m_{1}-1} u_{t=0}, \cdots, u_{m_{1}-1 \mid t=0}$ are uniquely determined and $u_{l \mid t=0}=0$ for $l \geqq m_{1}$. Put $G=\sum_{l=0}\left(u_{l \mid t=0}\right)(\log t)^{l}$, then there holds $Q G=t F$ for some $F \in L_{m_{1}-1}$. By $U=G-t V$, the equation $Q U=0$ is equivalent to $Q[1] V=F . \quad$ By the result of (i), this equation has a solution $V=\sum_{l=0}^{m_{1}-1} v_{l}(\log t)^{l}+\sum_{l=1}^{s-m_{1}} v_{m_{1}+l-1} t^{\lambda_{m_{1}+l}-1}(\log t)^{m_{1}+l-1}$. We can take $\left\{v_{l} ; l=0,1, \cdots, s-1\right\}$ such that the map $\alpha \mapsto\left(v_{0}, \cdots, v_{s-1}\right)$ is continuous linear. By the induction hypothesis, $\operatorname{Ker}^{\sim} Q[1]$ is parametrized by $C^{\infty}\left(\boldsymbol{R}^{n}\right)^{r^{\prime}}$, where $r^{\prime}=\sum_{j=2}^{d} r_{j}$. Hence, we get (ii) by the argument similar to that in the proof of Theorem 1.3.

\section{References}

[1] Baiocchi, C. and Baouendi, M.S., Singular evolution equations, J. Functional Analysis, 25 (1977), 103-120.

[2] Baouendi, M.S. and Goulaouic, C., Cauchy problems with characteristic initial hypersurface, Comm. Pure Appl. Math., 26 (1973), 455-475.

[3] Blum, E.K., The Euler-Poisson-Darboux equation in the exceptional cases, Proc. Amer. Math. Soc., 5 (1954), 511-520. 
[4] Bragg, L.R., Exceptional Cauchy problems and regular singular points, Applicable Analysis, 14 (1983), 203-211.

[ 5 ] Diaz, J.B. and Weinberger, H.F., A solution of the singular initial value problem for the Euler-Poisson-Darboux equation, Proc. Amer. Math. Soc., 4 (1953), 703-715.

[6] Hasegawa, Y., On the initial-value problems with data on a characteristic hypersurface, J. Math. Kyoto Univ., 13 (1973), 579-593.

[ 7 ] Lewis, J. E. and Parenti, C., Abstract singular parabolic equations, Comm. in Partial Differential Equations, 7 (1982), 279-324.

[ 8 ] Tahara, H., Cauchy problems for Fuchsian hyperbolic partial differential equations, Proc. Japan Acad., 54 (1978), 92-96.

[9] - Fuchsian type equations and Fuchsian hyperbolic equations, Japan. J. Math., New Ser. 5 (1979), 245-347.

[10] — Singular hyperbolic systems, III. On the Cauchy problem for Fuchsian hyperbolic partial differential equations, J. Fac. Sci. Univ. Tokyo Sect. IA, 27 (1980), 465-507.

[11] Weinstein, A., Sur le problème de Cauchy pour l'equation de Poisson et l'equation des ondes, C.R. Acad. Sci. Paris, 234 (1952), 2584-2585. 
